Gut, 1960, 1, 205.

\title{
GASTRIC CYTODIAGNOSIS: A REVIEW AND APPRAISAL
}

\author{
BY \\ D. D. GIBBS \\ From the London Hospital
}

This paper presents a careful appraisal of the technique of gastric cytodiagnosis, and sets out the criteria for diagnosis necessary to avoid false positive results.

By staining air-dried blood films with new dyes, Ehrlich (1891) showed the value of morphological criteria in accurate diagnosis of many haematological disorders. Similarly, in the field of exfoliative cytology, it was the development of a new staining technique by Papanicolaou (1942) that provided fresh opportunities and a stimulus for investigation, and the resulting practical application of a new aid in diagnosis. Weiss (1959) briefly surveyed the potentialities and limitations of cytodiagnostic methods; it is particularly the cytology of vaginal secretions and sputum that has become a routine and valuable test for cancer in many laboratories.

Like the cytology of other organs, gastric cytodiagnosis has a venerable history, with pioneer investigations by several continental pathologists at the end of the last century. The cytology of gastric washings has been the subject of much wider interest in the past 12 years. Nevertheless, the correct place of the investigation in patients suspected of gastric cancer, let alone as a screening method in patients with vague or minimal symptoms, remains uncertain. Unfortunately, many papers giving impressively high percentages of "true" positive diagnoses from washings done on patients later shown to have gastric cancer, omit information on the particular character of the growths, which are often diagnosed more quickly and easily by other methods. Some assessment of the contribution that gastric cytology can offer, in the context of information derived from clinical, radiological, and gastroscopic approaches, has recently been published (Burnett, MacFarlane, Scott Park, and Kay, 1960).

It may be predicted that the rewards of gastric cytology will be threefold. First, as impressively shown by Schade (1958, 1960), the method may provide the only means of diagnosis in certain "surface carcinomas" that are confined to the mucosa. Secondly, the presence of malignant cells in gastric washings will provide a firm diagnosis in a proportion of cases in which the radiological appearances are suspicious or inconclusive; but it will be seen that less reliance can be attached to negative washings in favouring benign lesions. Thirdly, cytological examination is useful, both as a curb to unnecessary investigation and as a guide to management in some patients with advanced malignant disease of the stomach.

Several investigators have advocated rather elaborate methods of collecting gastric material, or favour the use of special apparatus to scrape the mucosal surface of the stomach. In fact, it is likely that simple gastric lavage, carried out after an overnight fast, usually proves as rewarding as more complicated techniques and causes a minimum of discomfort to the patient. Details of the simple method of collection, and of Papanicolaou staining techniques are readily available (Staff of the Vincent Memorial Hospital, 1950; Schade, 1960). In brief, the stomach must be cleared of all food remnants; saline washings are then made through an intragastric rubber tube with multiple perforations with the patient in various positions; the aspirate is centrifuged with a minimum of delay, and the deposit is smeared and put into fixative while wet; the slides are carried through haematoxylin, followed by Papanicolaou's alcoholic staining solutions, and are finally cleared and mounted.

For malignant cells to be available it is naturally conditional that the growth should involve the mucosal lining of the stomach. It is rare for a localized submucosal cancer to be present, or for carcinomatous infiltrations to remain entirely deep to the mucosa. The presence of large necrotic tumours, or of pyloric obstruction, is much more commonly responsible for "false negative" results. In these circumstances it may not be possible to 
collect aspirates that contain recognizable malignant cells.

Even with experience, the scanning and interpretation of smears is exacting in time. In practice it is found that at least three hours should be set aside for the collection, preparation, and examination of material from a patient, and longer may be required before a search is pronounced negative with certainty.

It is unlikely that cytodiagnosis will be recommended for the routine diagnosis of gastric cancer but, for the investigation of selected patients, it will undoubtedly find a place. An intention of this article is to give an idea of its possibilities and limitations; it is hoped that a judgment will be possible on the validity of some of the evidence used in reaching cytological diagnoses.

\section{The Origin of Cells in Gastric Washings}

Cells collected from the stomach present a greater challenge to the resourcefulness of the exfoliative cytologist than material from other sites, by virtue of the fact that the stomach acts as a temporary reservoir for secretions from various sources. Cells may originate from respiratory, oral, nasal, or oesophageal mucous membranes; they exfoliate from the gastric mucosa sparsely in health, but profusely in various local and diffuse abnormalities; or, rarely, they may be derived from the duodenum or biliary tract, carried by the retrograde flow of juices.

Few attempts have been made to correlate the cytological appearances seen in smears with the histological sections of "parent" tissue taken from specimens after operation. Schade (1959) has made a contribution to this basic task.

\section{Available Illustrations of Gastric Cytology}

Attention will be drawn to the publications on gastric cytodiagnosis, in which helpful illustrations accompany the texts.

The Cytologic Diagnosis of Cancer, by the staff of the Vincent Memorial Hospital (1950), illustrates effectively many of the characteristic appearances in exfoliative cytology. Low-power views are given, with parallel illustrations in high magnification, of particular groups of cells. A lack of clarity in reproducing the photomicrographs is compensated for by drawings of the same fields. There is little consideration given to the many deviations from normal that may be seen in benign conditions, and only a few representative malignant cells from the stomach are depicted.

Papanicolaou's Atlas of Exfoliative Cytology (1954, Supplement 1956) offers a major source of reference on the subject, and at present acts as the archives for illustrations of cytological material. Kodachrome photomicrographs and drawings in colour are portrayed. The drawings are beautifully executed and minute attention has been given to detail. It is inevitable, however, that the separation of the cells from their backgrounds and the addition by the artist of a dimension of depth, should tend to give a slightly artificial, even stylized quality, to some of the drawings. There are no less than 132 illustrations of the cytology of the digestive system. Additions have been made as new material has become available, with the result that there is no systematic presentation. As most of the material illustrated is derived from specimens obtained by abrasive gastric balloons, there has been comparative neglect of the non-malignant cytology of the stomach.

Henning and Witte (1957) illustrate their Atlas der gastroenterologischen Cytodiagnostik with an excellent series of photomicrographs. Their material was collected by a special device, the "Zelltupfsonde", enabling cells to be obtained by sponging the mucosa, and their studies are particularly valuable in showing the cellular changes that take place in atrophic gastritis. They also depict chief or parietal cells, which appear to resist the effects of simple lavage, for they are extremely rare in aspirates.

Richer (1956) has written a valuable and critical account of his experience of gastric washings in diagnosis, and he includes 12 photomicrographs in his paper. It is particularly noteworthy that these include cells showing atypical but non-malignant changes, which he found difficult to interpret with certainty from some patients with atropic gastritis or healing gastric ulcers.

Bruinsma (1957) reports his studies on a small series of patients with carcinoma of the stomach, in whom he used the "gastric balloon". The accompanying photomicrographs, especially those in colour, are realistic and of high quality, the majority representing malignant cell groups.

Schade (1960) has published a treatise on gastric cytology of great importance, in which he traces the historical development of the investigation, mentions the various techniques of collection that have been advocated, and presents the results of his experience derived from cytological studies on 2,443 patients since 1954. By the use of simple lavage and examination of smears he has obtained highly impressive results, claiming an overall accuracy of $96 \%$ in a group of 522 of these patients in whom the diagnosis was proved by laparotomy or resection. There were a further 36 patients in whom the diagnosis was established at operation, but whose gastric aspirates had been deemed unsuitable for 
cytological examination. Twenty-four of these had carcinoma. It might be presumed that some had advanced growth or obstruction, in which circumstances reasonably clear washings are notoriously difficult to obtain. In the 264 patients in whom a benign condition was found at resection (mainly peptic ulceration), and from whom suitable aspirates were obtained, 13 "false positives" occurred, constituting about $5 \%$ of the total. The greater percentage of "false positive" diagnoses in the benign group than "false negative" results in the cancer group is explained by Schade on the basis of atypical but non-malignant epithelium, in mucosa surrounding ulcers.

The most remarkable feature of Schade's work is the report of 16 surface cancers in patients not suspected of malignancy on clinical or radiological grounds; the histology of several of these tumours is illustrated convincingly. These patients came to operation, apparently, only as a result of positive cytodiagnosis, obtained during a survey investigation on gastritis. It is implied that such localized early carcinomas may come to the attention of clinicians on account of symptoms produced by chronic gastritis, which is thought frequently to precede rather than merely accompany malignant change.

The choice and quality of photomicrographs in Schade's book should receive comment. To those with some experience of exfoliative cytology, most of the illustrations of malignant cells in smears will carry conviction. They convey, particularly, those criteria of malignancy that apply to abnormal arrangements and relationships within cell clusters, but there is often lack of clarity in displaying the inherent features of malignancy of the cells. It is disappointing not to have specific representation of some of the cells that led to "false positive" reports. It is in this group that the criteria of malignancy are put to their most severe test, and with the knowledge of the final histological diagnosis it would have been possible to appreciate some of the difficulties that may arise in making interpretations.

\section{Characteristics of Cells Collected from the STOMACH}

Classification of cell types encountered must be based on the probable sites of origin of cells that have been shed into the stomach, though it will be realized that the major relevance of special features present in the various cells lies in the way in which these resemble or differ from the cytological characteristics of malignancy. It is with this in mind that the descriptions are presented. Schade (1960) has correctly stressed the additional importance of the "background" in smears-blood, leucocytes, goblet cells, meat fibres, bacteria - and there is no doubt that such features can provide valuable supporting evidence in a cytological opinion.

SQuamous Epithelial Cells.-Squames from oral and oesophageal epithelial surfaces are nearly always the most prominent cells in gastric washings. A simple division into superficial squamous cells and parabasal cells is sufficient.

(1) Superficial squamous cells are easily recognized (Fig. 1). They are large and flat, with sharp, rather squared-off edges; the cytoplasm is usually pink, but may be basophilic and take up a light blue tint; granular and hyaline material is often present in the cytoplasm; nuclei are oval, centrally placed, and stain blue, or become small and densely pyknotic in the most superficial cells.

(2) Parabasal cells (Fig. 2) are comparatively infrequent but may occasionally lead to confusion. The cytoplasm is a fairly deep blue; the nucleus is usually eccentric, and has a sharp nuclear membrane. The nuclei may be hyperchromatic and contain granular chromatin and one or more prominent nuclei, thus reflecting an "active" appearance, due to the fact that they are making good the loss, from wear and tear, of the superficial layers of epithelium.

Cells from Respiratory EpIthelium.-Ciliated columnar epithelial cells, which range from tall and delicate structures, to plump, broad, multinucleated forms, are desquamated from bronchial or nasal mucous membranes. They are distinctive so long as they remain well preserved (Figs. 3 and 4). They are not very common in gastric washings, but may be carried to the stomach in swallowed sputum, or by the passage of a naso-oesophageal tube. The nuclei may be slightly irregular, densely staining and overlap one another. If these cells are present in clumps and are devoid of most of their cytoplasm, they may arouse false suspicions of malignancy.

Histiocytes or Macrophages.-(1) Respiratory histiocytes (Fig. 5) are constantly present in sputum and therefore frequent in gastric washings. They are usually easily identified and as a rule distinguishable from the macrophage elements of the gastrointestinal tract. They have a round or slightly oval outline; cytoplasm is greeny-blue, foamy, and typically, though not constantly, contains black particles of carbon; nuclei are purplish blue, oval or kidney shaped, and placed eccentrically. Cells may contain two or more nuclei. The cells themselves are often to be found in ranks, strung out in strands of mucus. 


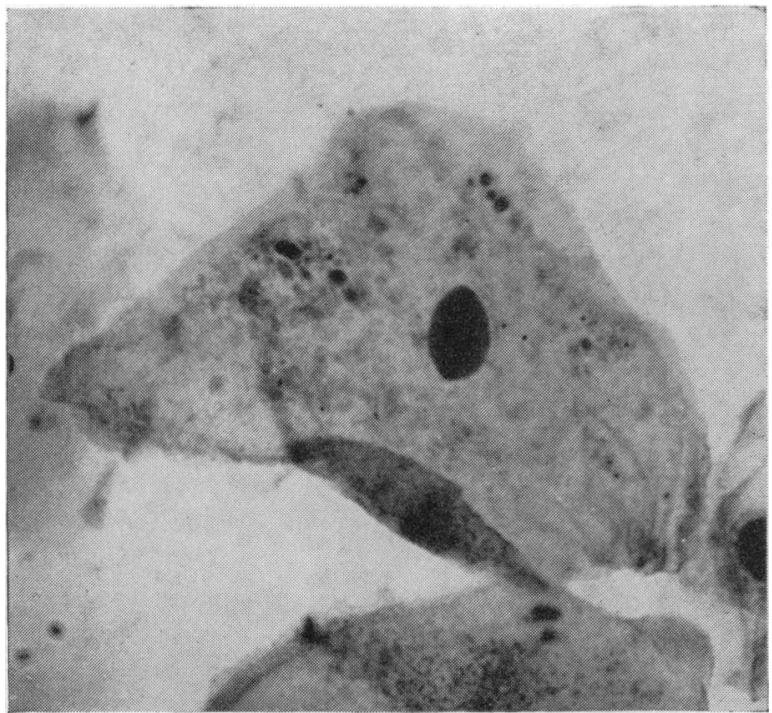

Fig. 1.-Squamous epithelial cells from superficial layers.

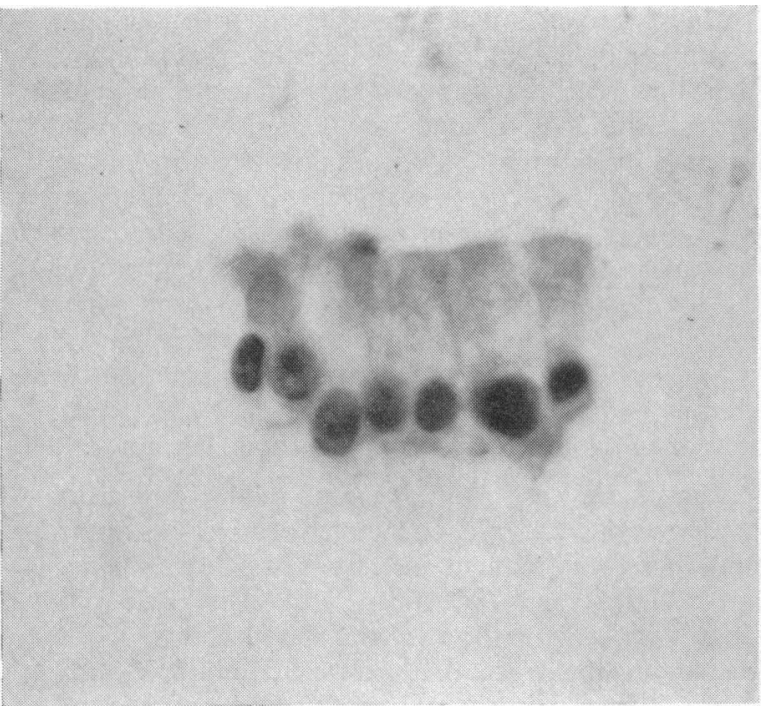

FIG. 3.-Respiratory epithelium. Well-preserved, columnar, ciliated cells.

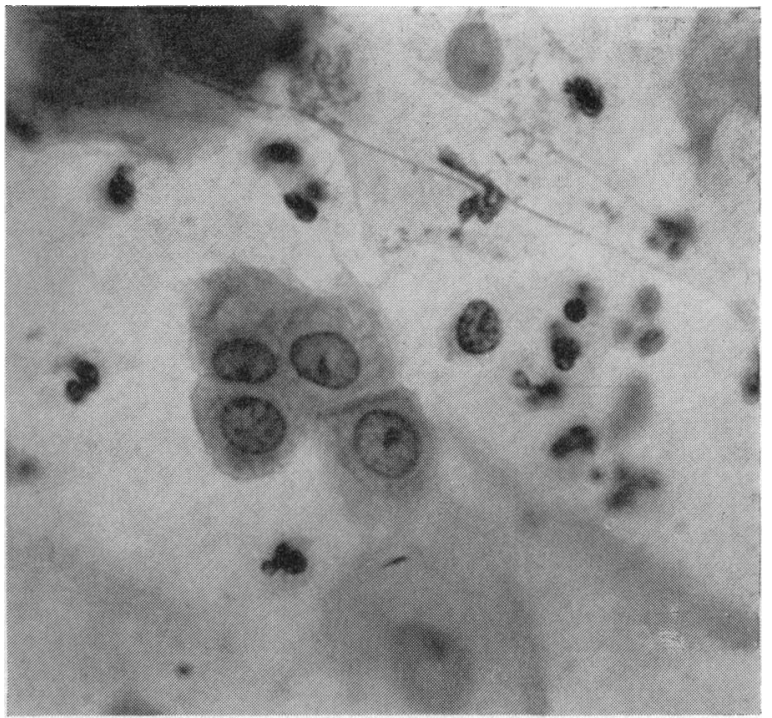

FIG. 2.-Squamous epithelial cells: clump of four cells from basal layers, with superficial squames. Polmorphs. Bacteria.

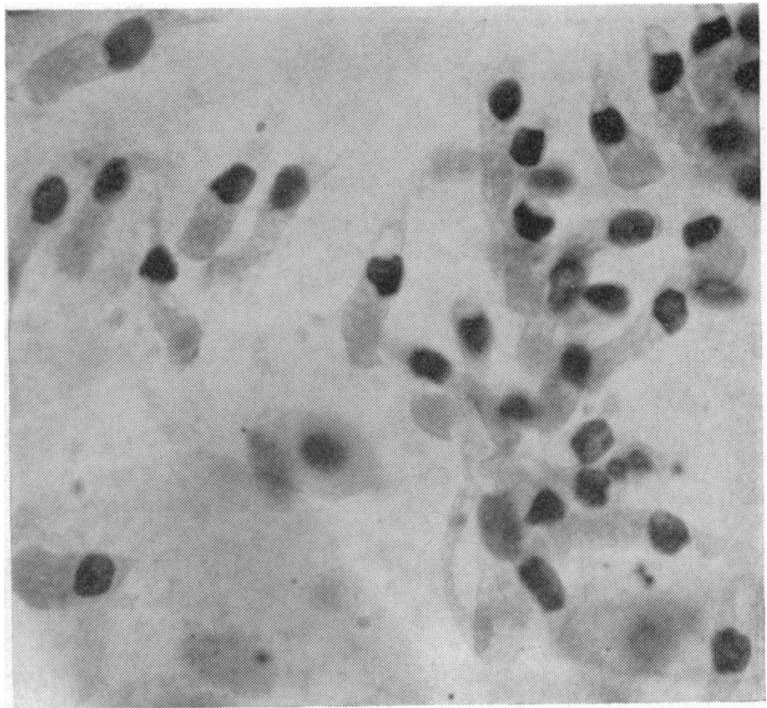

FIG. 4.-Respiratory epithelium.

The approximate magnification of cells represented in the photomicrographs is $\times 850$. 


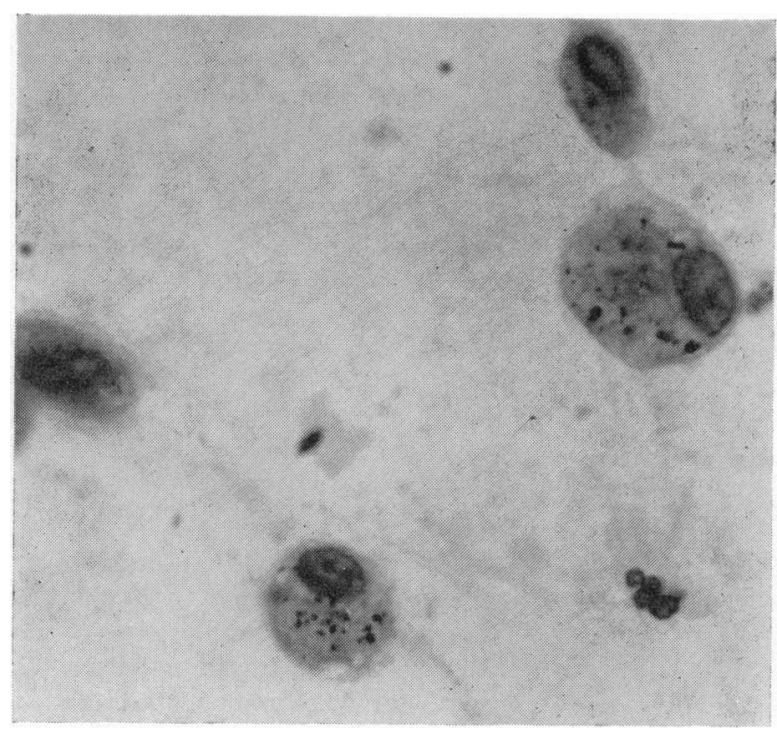

Fig. 5.-Histiocytes in swallowed sputum; "dust cells", with carbon particle inclusions.

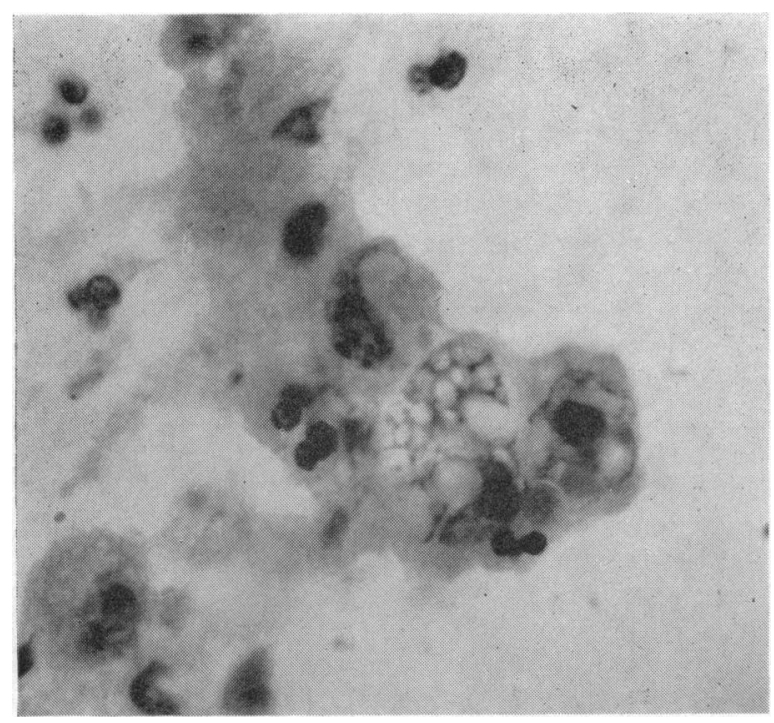

Fig. 7.-Histiocytes from stomach. Accretion of cells with ingested refractile material.

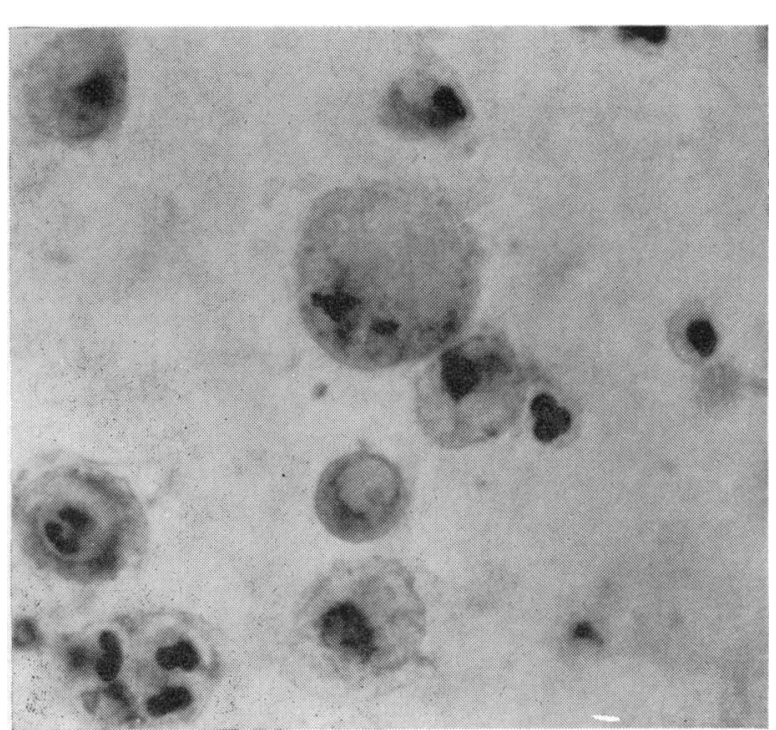

FIG. 6.-Histiocytes from stomach; droplet and leucocyte inclusions.

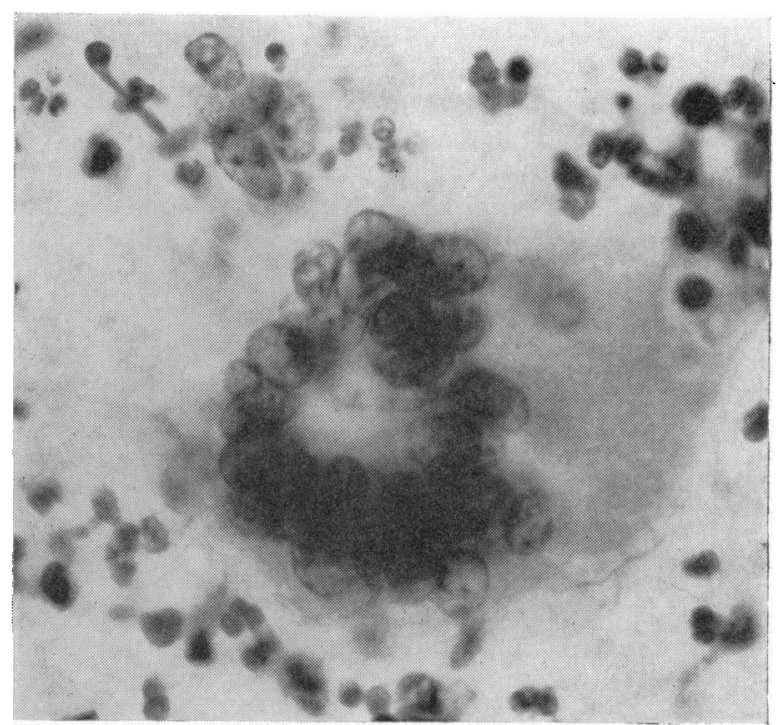

Fig. 8.-Multinucleated histiocyte. "Foreign body giant cell." 
(2) Histiocytes of gastrointestinal origin (Fig. 6) are not normally seen, but may appear in large numbers in washings from patients with gastric ulcer, atrophic gastritis, or carcinoma. Their general configuration is the same as those of respiratory origin, but differences in detail are usually recognizable. The cell borders are often indistinct; the cytoplasm is frequently foamy, sometimes containing large vacuoles, but lacks carbon particle inclusions. The staining property of the cytoplasm is variable, and it does not assume the greeny-blue hue usual in respiratory histiocytes. Nuclei tend to have less sharply defined borders and to be less regular in shape. Phagocytosis of leucocytes is very common, and the histiocytes, at times, appear crammed with polymorphonuclear remnants, which tend to displace and distort the nuclei.

Giant Histiocytes.-Occasionally foreign body giant cells (Fig. 8) are to be found in smears from gastric washings, usually in the presence of extensive gastric tumours. They contain many nuclei, which are often disposed peripherally.

Cells Derived from Blood.-(1) Red blood cells are common in saline gastric washings, and it is important to realize that visible blood in the centrifuge deposit is a fairly frequent finding in non-malignant conditions. This, of course, contrasts with the frequently sinister import of blood in a gastric test meal; the trauma from jets of saline in a washing often seems sufficient to produce mucosal haemorrhage, in the absence of malignant disease, though the mucosa is obviously abnormal in many non-malignant conditions.

(2) Polymorphonuclear leucocytes are sparse in the normal fasting stomach, but are often present as a dense background in smears from patients with carcinoma, ulcer, or gastritis. They are to be seen infiltrating the abnormal mucosa in atrophic gastritis, and enclosed in macrophages, and sometimes in malignant cells (Fig. 18). Their state of preservation varies and probably depends on the degree of acidity. Occasionally clumps of purple dots are present, and represent fragmented nuclear lobes.

(3) Round Cells.-Lymphocytes seldom predominate in smears, but their presence, in conjunction with abnormal forms, might suggest the diagnosis of lymphosarcoma of the stomach (Klayman, Kirsner, and Palmer, 1955). Plasma cells are common in atrophic gastritis, and they are easily recognized by their eccentric, "cartwheel" nuclei, and fairly abundant, often reddish cytoplasm.

Cells from Gastric Epithelium.-The columnar epithelium which lines the stomach does not exfoliate readily in health, and the cells when present show considerable constancy of shape and size, though their states of preservation vary. Large groups of cells are usually seen end on. They then show polygonal outlines, most cells having distinct borders, and particularly from the basal surface, they show a honeycomb-like appearance (Fig. 9). Cells scattered in small groups or singly often appear from their lateral aspects, showing rectangular outlines, faintly blue cytoplasm, and unipolar nuclei which contain finely granular material. The cells are particularly susceptible to the cytolytic action of gastric juice, which often denudes them, so that only cytoplasmic remnants cling to the nuclei, or only naked nuclei remain.

Striking changes in the cells from the stomach with atrophic gastritis may appear, and it is these that are most likely to cause diagnostic difficulties, by mimicking those seen in true malignant cells. Although the clinical relevance of atrophic gastritis may be argued, there has been greater appreciation of the frequency of the histological change, since the work of Doig and Wood (1958), using the flexible biopsy tube. Though gradations exist, a distinction can be made between the three histological pictures of superficial and atrophic gastritis, and complete gastric atophy. Atrophic gastritis may itself be "zonal", near lesions such as gastric ulcer or carcinoma, or near an anastomotic stoma; or it may be "diffuse". No real estimate of the extent of the mucosal change can be gauged from gastric washing. From the affected areas the superficial layer of epithelium exfoliates profusely, the cells often appearing in syncytial sheets. The cells are plumper and more circular than normal, and show varying degrees of anisokaryosis, nucleolar prominence, and overlapping of nuclei (Figs. 10, 11, and 12). In addition, goblet cells, each containing a distended vacuole that may compress the nucleus, are often present, and indicate that areas of mucosa have altered to the intestinal pattern. Abundant polymorphs, some of which permeate the abnormal epithelium, macrophages and plasma cells, often contribute further to the picture.

Brief reference will be made to alterations in the gastric epithelium observed in patients with pernicious anaemia. It is known that there is nuclear enlargement of cells from several epithelial surfaces (Boddington and Spriggs, 1959). Rubin (1955) mentioned two distinct types of gastric epithelial cell, the "large bland columnar cell" and the "active cell". The active cells are well preserved and show chromatin clumping and large nuclei (Fig. 15), but it is now clear that they are not confined to patients with pernicious anaemia, and may be present with chronic atrophic gastritis (Henning and Witte, 1957). 


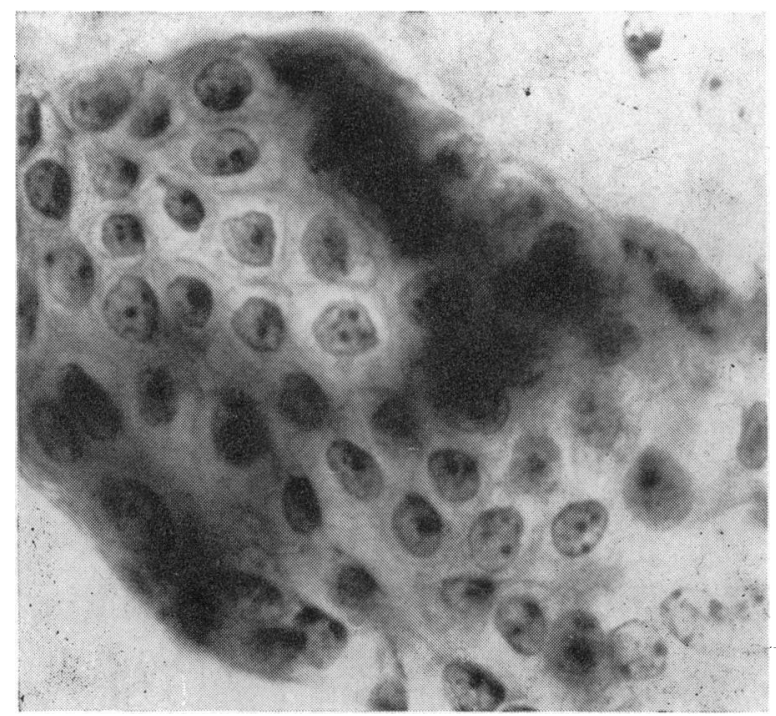

FIG. 9.-Gastric surface epithelium. "Honeycomb" appearance with clear definition of cell borders.

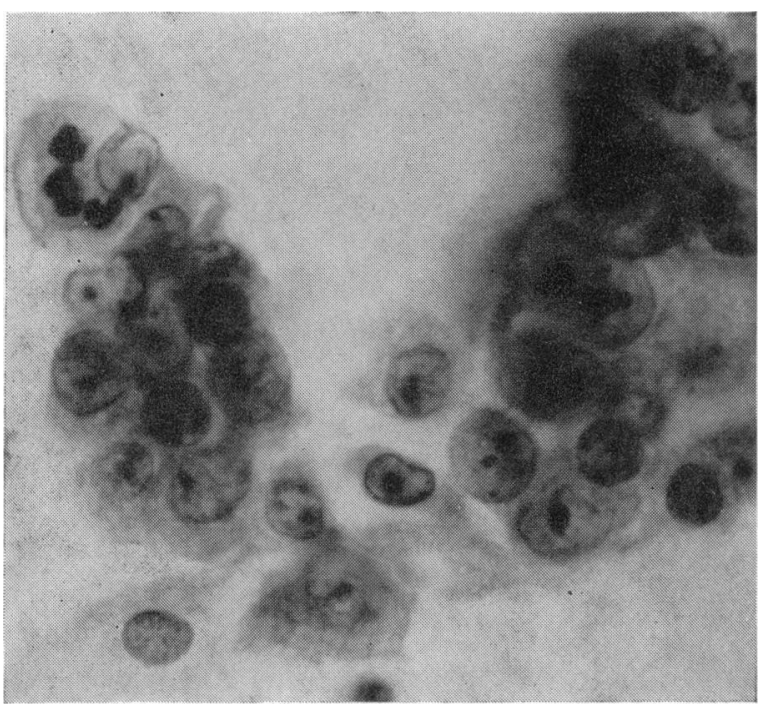

FIG. 11.-Atrophic gastritis. Nuclear variation in shape and size, "overlapping", nucleolar prominence. Leucocyte inclusions in epithelium.

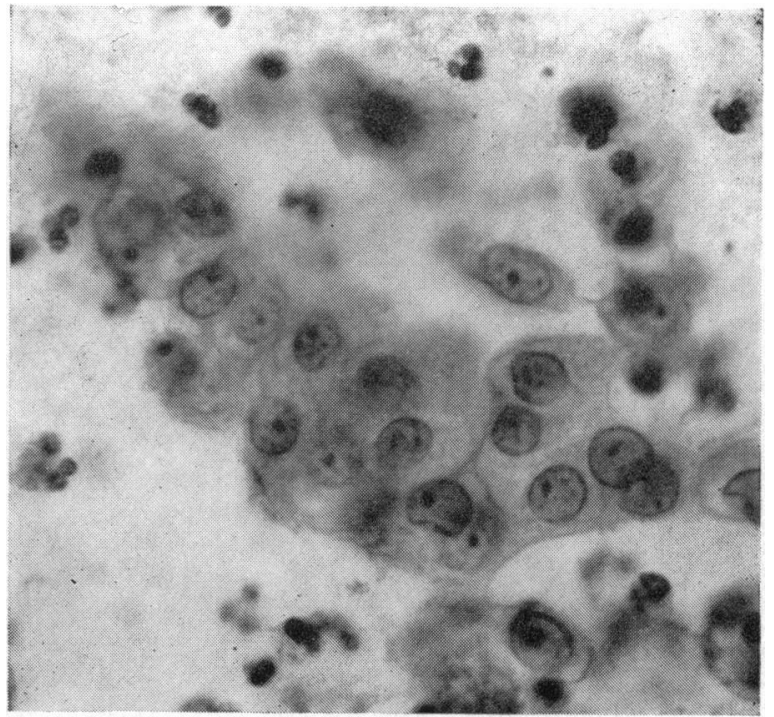

FIG. 10.-Atrophic gastritis. Syncytial appearance of mucosal cells.

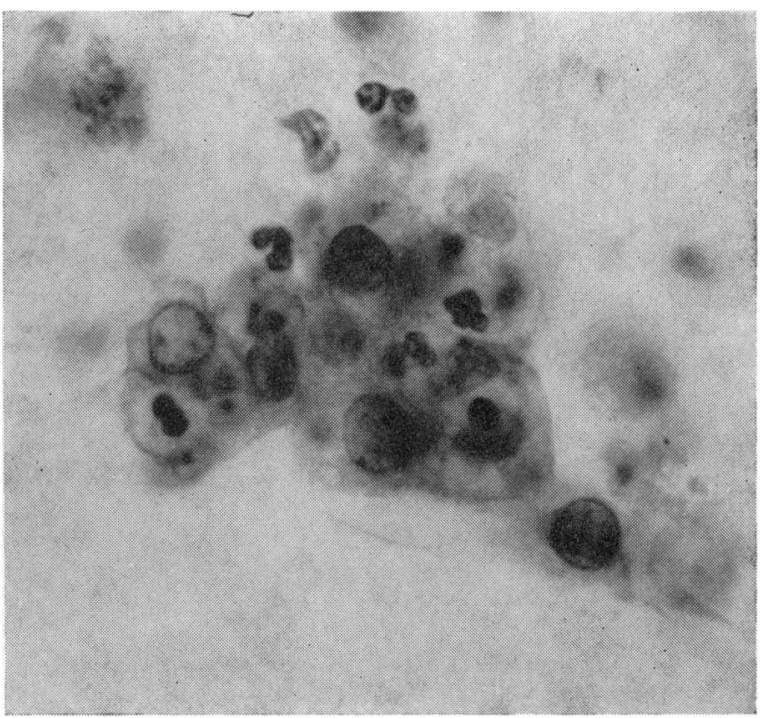

Fig. 12.-Atrophic gastritis. Clump of epithelial cells with indistinct borders and polymorph inclusions.

The approximate magnification of cells represented in the photomicrographs is $\times 850$. 


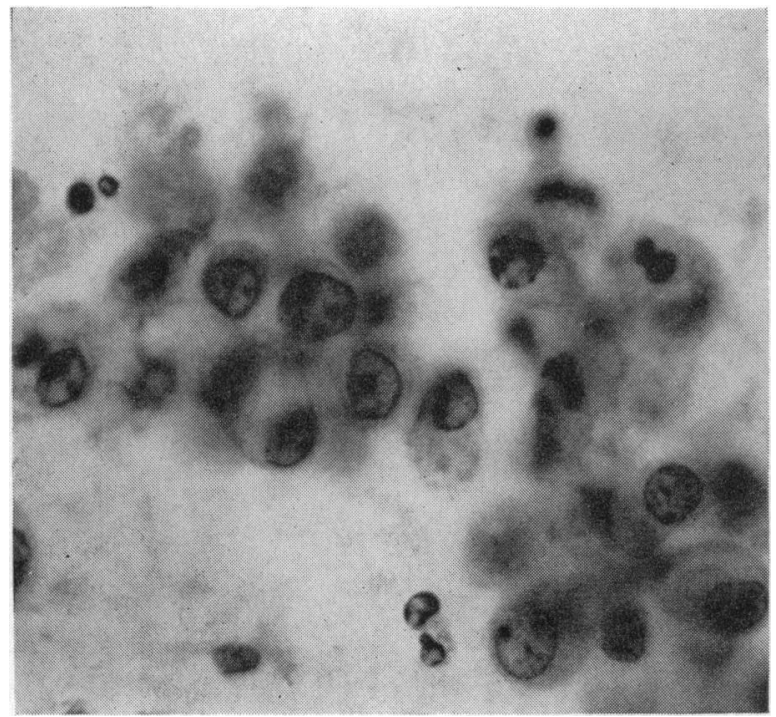

FIG. 13.-Gastric epithelial cells showing nuclear irregularity, nucleolar prominence, and leucocyte inclusions.

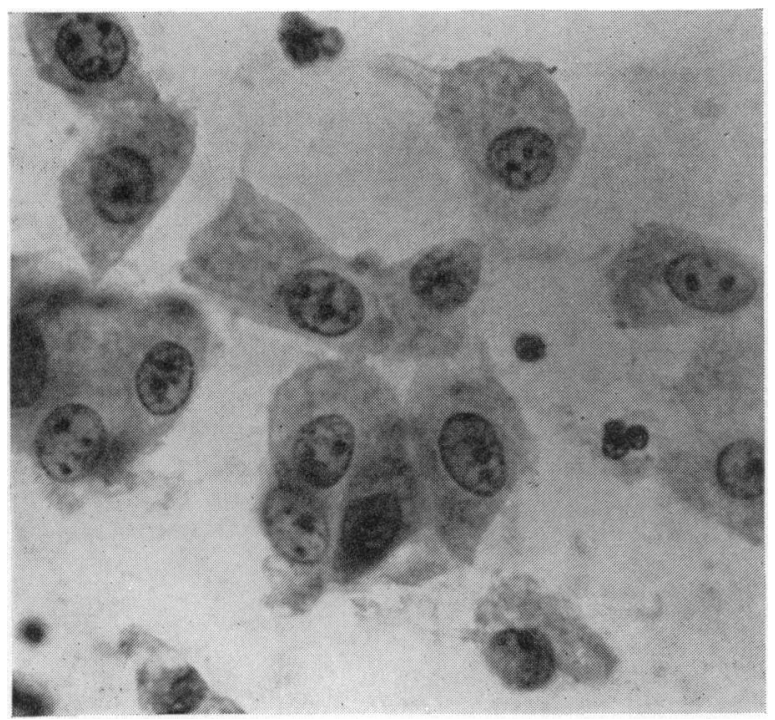

FIG. 15.-Gastric columnar cells which are plump, contain vacuolated cytoplasm, and have "active" nuclei. Patient had achlorhydria; laparotomy was negative.

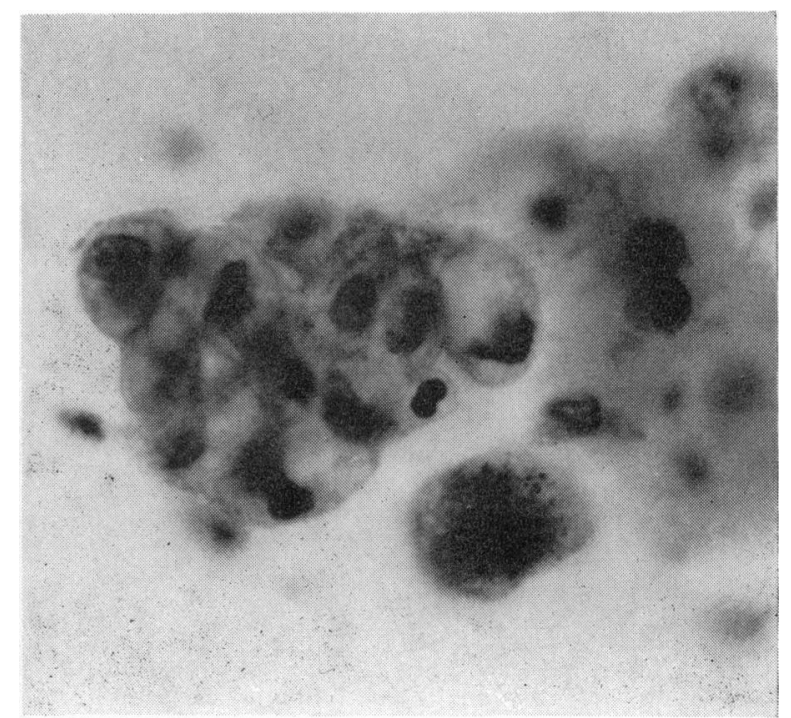

FIG. 14.-Cluster of closely packed gastric epithelial cells, two showing features of "goblet cells". No cancer at operation.

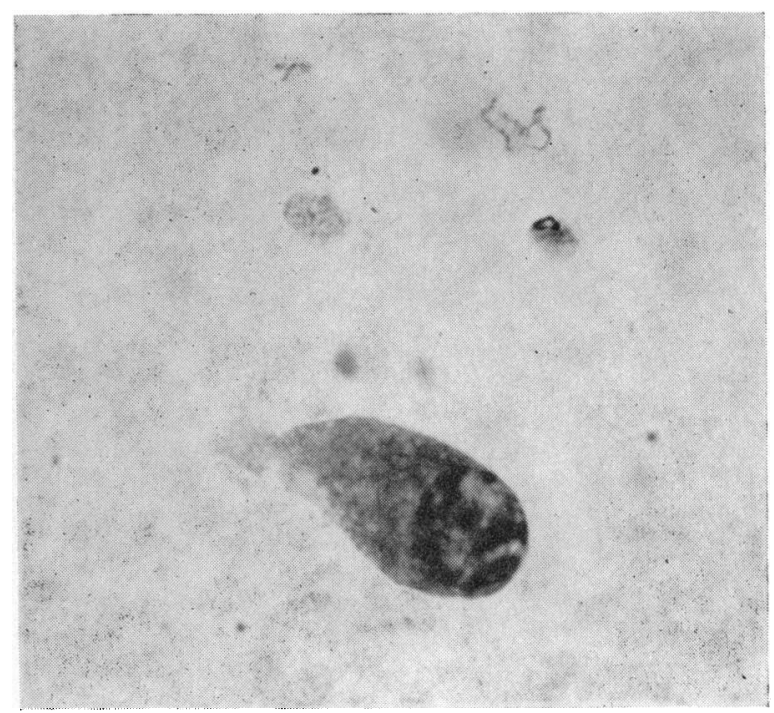

Fig. 16.-Single columnar carcinoma cell; impressive clumping of chromatin in the nucleus.

The approximate magnification of cells represented in the photomicrographs is $\times 850$. 


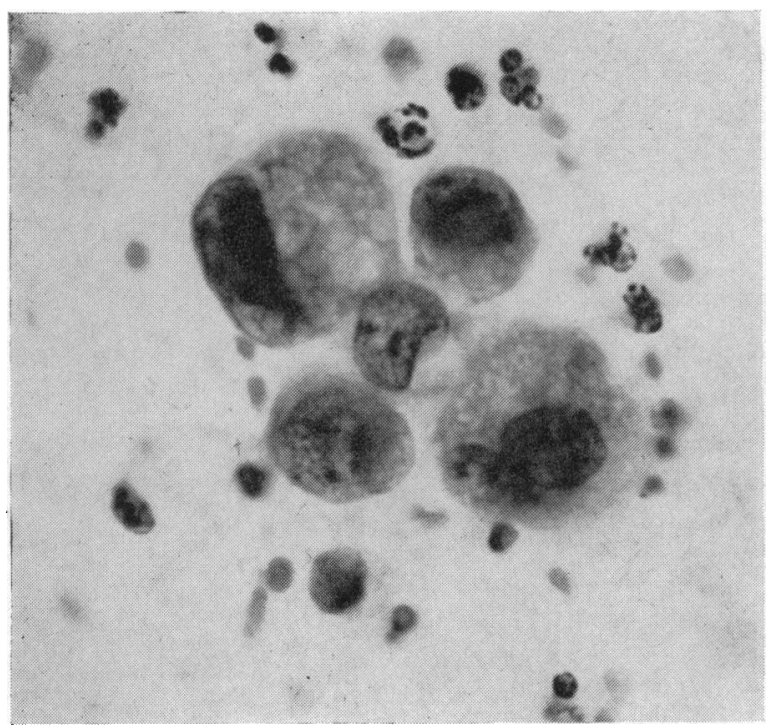

Fig. 17.-Group of carcinoma cells from a patient with "leather bottle" stomach. Note large size of cells, impressively vacuolated cytoplasm, and gross nuclear abnormalities.

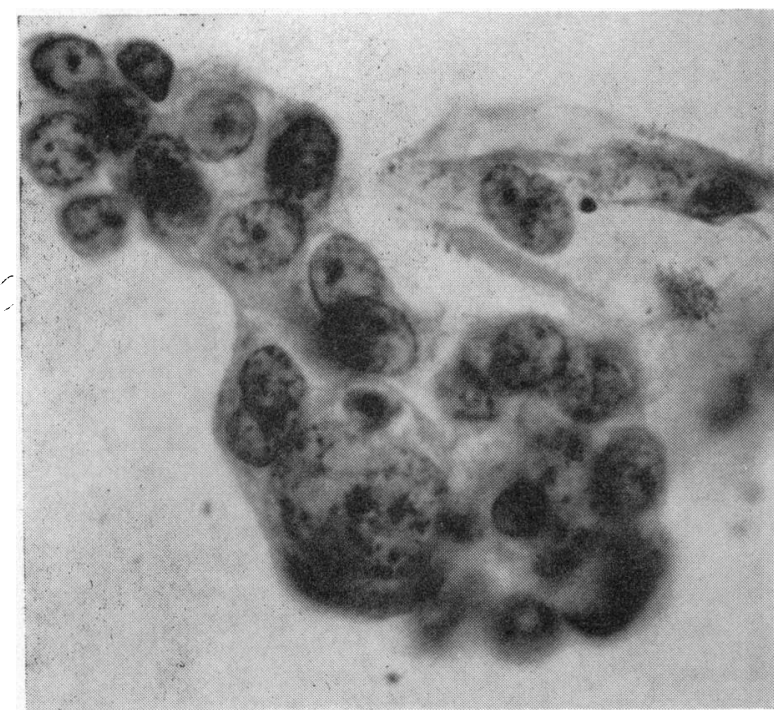

Fig. 19.-Undifferentiated gastric carcinoma cells, including a giant malignant nucleus.

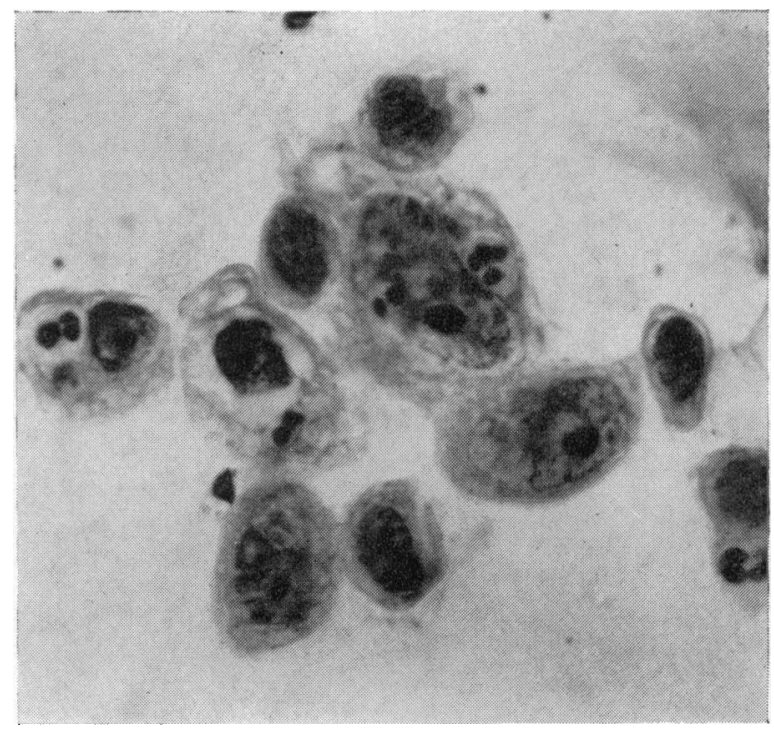

FIG. 18.-Undifferentiated gastric carcinoma cells. Note giant nucleoli and cytoplasmic inclusions of leucocytes.

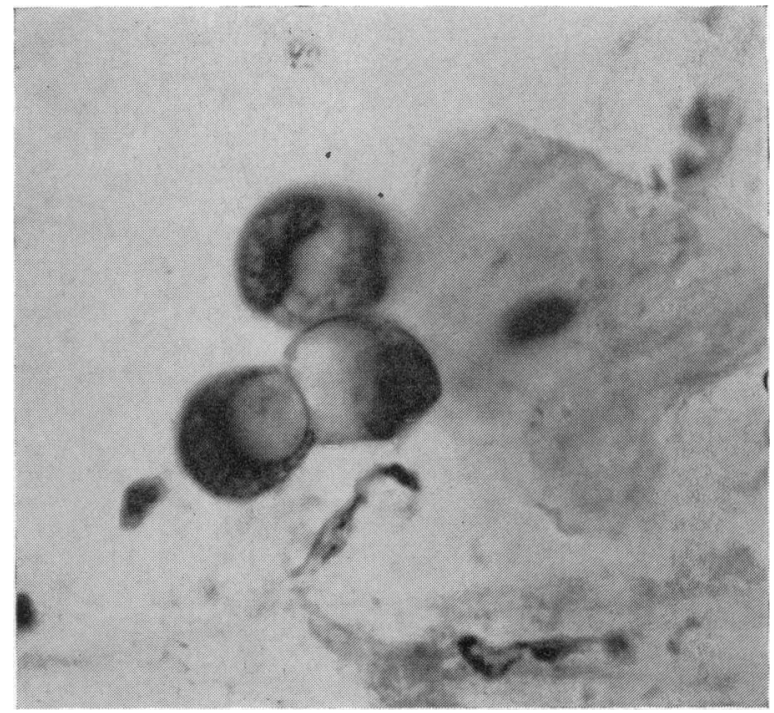

FIG. 20.-Mucus-secreting carcinoma cells. 


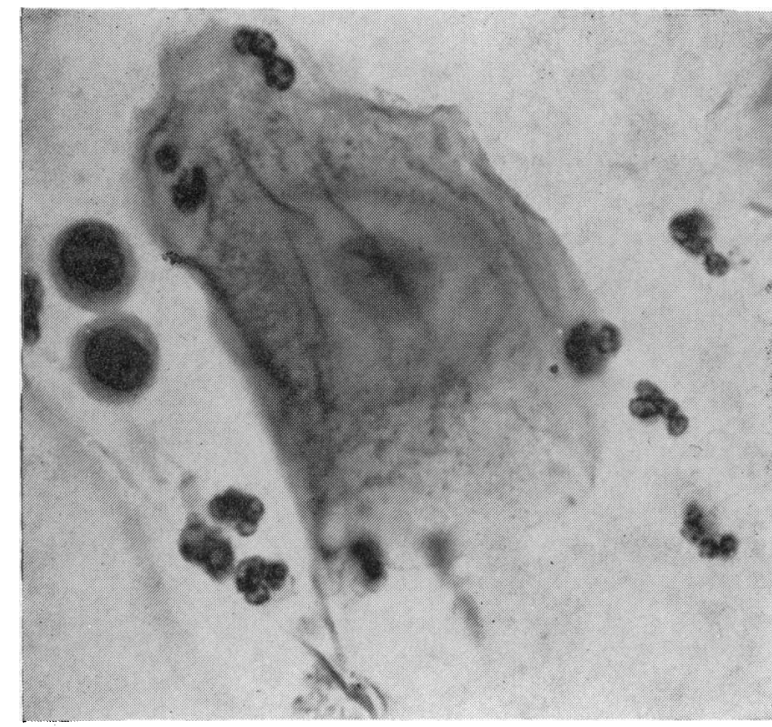

FIG. 21.-Two small carcinoma cells; superficial squamous cell; polymorphs. Note abnormal nucleo-cytoplasmic ratio and hyperchromasia shown in malignant cells.

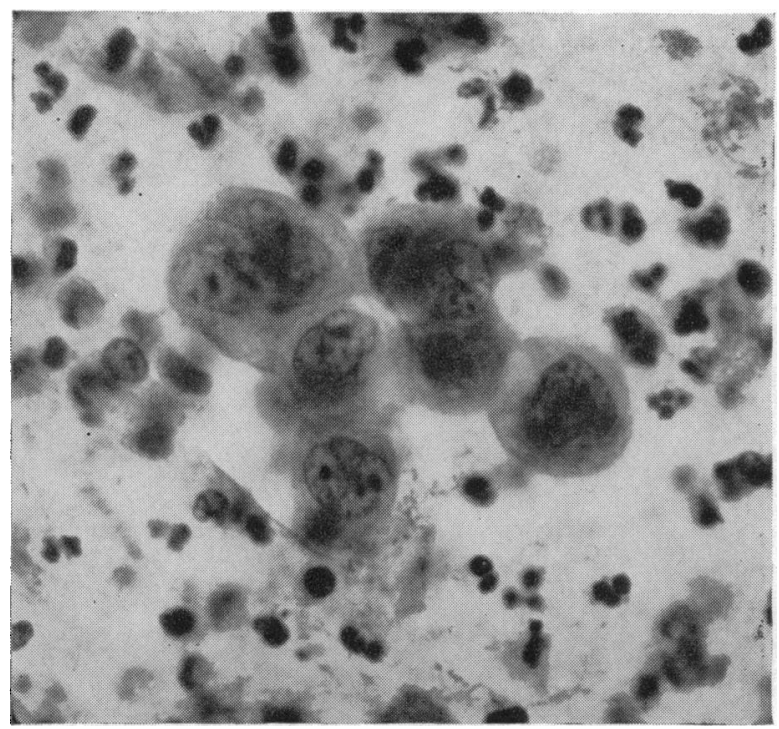

FIG. 23.-Group of carcinoma cells against a dense background of polymorphs. Note grossly abnormal nuclear features, including lobulation indentation and multiple nucleoli.

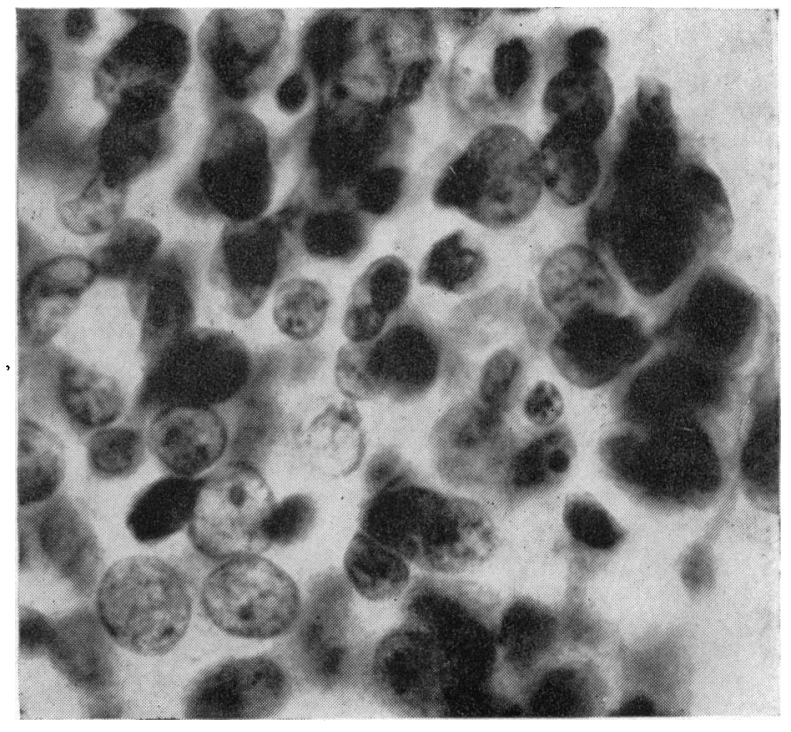

Fig. 22.-Sheet of undifferentiated carcinoma cells, illustrating nuclear variation and "overlapping". Operation specimen showed mucosal malignant change in a gastric polyp.

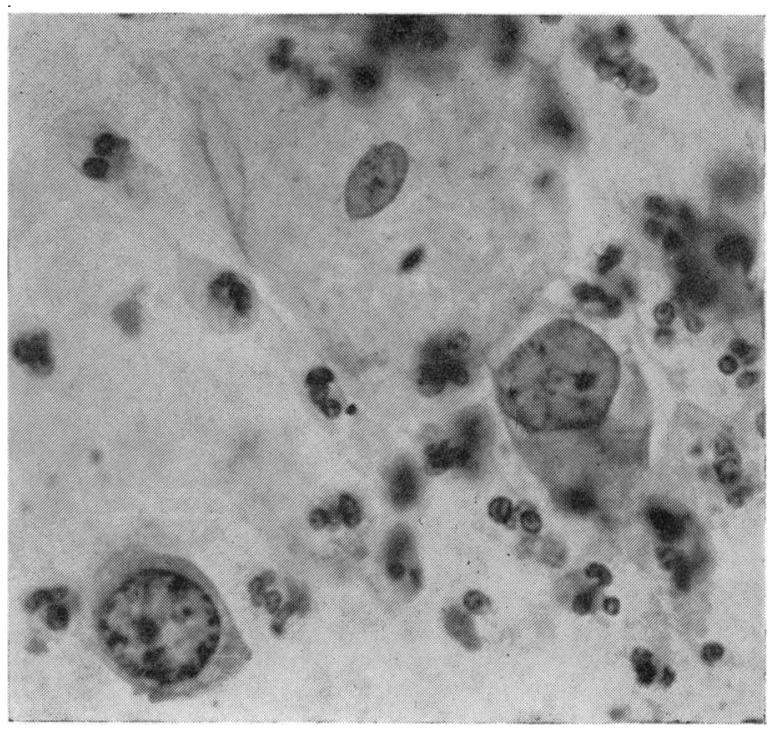

FIG. 24.-Two carcinoma cells; superficial squame; polymorphs. Note thickening of nuclear membrane in lower malignant cell. 
In giving cytological verdicts on patients with pernicious anaemia caution is clearly demanded, but the definite criteria of malignancy become no less reliable or valid.

\section{Cytological Criteria of Malignancy in Gastric CARCINOMA}

Papanicolaou (1954) gives the most useful general analysis of the criteria of malignancy in cytology; Schade $(1956,1960)$ lays emphasis on certain characteristics which have frequent application to material from the stomach. Some of the criteria will be recapitulated, but the most convincing persuasion may come from examination of the photomicrographs of malignant cells (Figs. 16 to 24). Cells vary both in number and prominence of malignant features that they display, and some of the non-malignant conditions that may cause confusion have been mentioned. The most notable are atrophic gastritis and healing gastric ulcers. A small number of cases show these borderline criteria, and the fact that difficulties exist should not be used as an argument against the validity of cytological diagnosis when cells show incontrovertible stigmata of malignancy. Extreme caution is necessary before pronouncing a doubtful smear as malignant; Richer (1956) wisely pleads: "Mieux vaut être timoré qu'imprudent."

The criteria of malignancy most frequently applicable in gastric cytodiagnosis are:

I. Nuclear changes suggesting malignancy:

(1) increase in nucleo-cytoplasmic ratio (Fig. 21); (2) hyperchromasia (Fig. 20); (3) abnormal chromatin patterns, including irregular clumping (Fig. 16), lobulation, indentation, and furrowing (Fig. 23); (4) nuclear gigantism (Fig. 19); (5) variations in nuclear size-anisokaryosis (Figs. 17 and 18); (6) enlargement, or an increase in number, of nucleoli (Figs. 18 and 23); (7) thickening of the nuclear membrane (Fig. 24); (8) crowding and overlapping of nuclei (Fig. 22).

II. Cytoplasmic changes (though providing little specific help in deciding on malignancy): (1) excessive vacuolation (Fig. 17) or mucus secretion, indicative of adenocarcinoma (Fig. 20); (2) leucocyte inclusions (Fig. 18).

III. Criteria derived from whole cells, and cell groups: (1) tendency to clump, without any regularity of pattern; (2) indistinct cell borders, or merging of cytoplasm (Figs. 18 and 19); (3) increase in size and anisocytosis (Figs. 17 and 18).

\section{CONCLUSION}

Clinicians frequently enquire as to the possible value of gastric cytology in investigating individual patients. Many papers have been written giving results of cytological investigations on series of patients with suspected or proven gastric cancer but, with few exceptions, there are inadequate data and illustrations to enable the reader to judge the basis upon which the cytological conclusions have been reached. Ultimately, the only valid test for the usefulness of gastric cytodiagnosis is to apply the investigation to patients suspected of carcinoma of the stomach, with adequate comparison of histological material obtained later from the same patients. Before undertaking such work, the investigator will want information on the reliability of the criteria that have been applied to exfoliated cells. There is little doubt that, once the cytologist has acquired sufficient special experience, the technique offers a valuable diagnostic approach which, though usually supplementary, may occasionally be vitally useful and provide an emphatically positive answer in the presence of equivocal, or rarely negative results, by other approaches. Firmly positive reports will probably prove possible, from single washings, in about $70 \%$ patients with gastric carcinoma. It is clear that undue reliance should not be placed on negative cytology in excluding carcinoma.

Many contributions on gastric cytodiagnosis have been marred by a significant number of false positive results, which can only mean an acceptance of criteria of malignancy that are insufficiently rigid, and a refusal to admit that, in a small proportion of cases, it is impossible to give a categorical answer.

An attempt has been made to survey briefly the subject of gastric cytodiagnosis. It will be inferred that the availability of the technique is not likely to be limited by any complexity of procedure, nor by reluctance of patients to accept it, but by the time and special experience available from a cytologist. Reference has been made particularly to those publications on gastric cytology that provide illustrations which are most likely to prove of value for comparative purposes. The remarkable reluctance of most authors to illustrate material that has led them to give false-positive diagnoses is mentioned. Certain cells encountered in gastric washings are described, and attention is given to the criteria used in the cytological diagnosis of gastric malignancy. Illustrations have been chosen to complement the descriptions and enable judgment to be made on the acceptability of the criteria.

I am grateful to the physicians and surgeons of the London Hospital for allowing me access to their patients. I am indebted to Mr. A. J. King, A.R.P.S., A.I.B.P., for the skill and meticulous care he has shown in preparing the photomicrographs. The cost of the photographs and 
materials was met by a grant from the London Hospital Research Fund.

\section{REFERENCES}

Boddington, M. M., and Spriggs, A. I. (1959). J. clin. Path., 12, 228.

Bruinsma, A. H. (1957). Thesis. Utrecht.

Burnett, W., MacFarlane P. S., Scott Park, S. D., and Kay, A. W. (1960). Brit. med. J., 1, 753.

Doig, R. K., and Wood, I. J. (1958). Modern Trends in Gastroenterology, 2nd Series, Chapter 13. Butterworth, London.

Ehrlich, P. (1891). Farbenanalytische Untersuchungen zur Histologie und Klinik des Blutes. Hirschwald, Berlin.
Henning, N., and Witte, S. (1957). Atlas der gastroenterologischen Cytodiagnostik. Thieme, Stuttgart.

Klayman, M. I., Kirsner, J. B., and Palmer, W. L. (1955). Gastro enterology, 29, 536.

Papanicolaou, G. N.'(1942). Science, 95, 438.

(1954; Supplement 1956). Atlas of Exfoliative Cytology. Harvard University Press, Cambridge, Mass.

Richer, C. (1956). Sem. Hop. Paris, 32, i, 958.

Rubin, C. E. (1955). Gastroenterology, 29, 563 .

Schade, R. O. K. (1956). Gastroenterologia (Basel), 85, 190.

(1958). Brit. med. J., 1, 743.

-(1958). Brit. med.J., 1, 743. 3,7

(1959). Acta cytol. (Chicago), 3, 7.

- (1960). Gastric Cytology. Arnold, London.

Staff of the Vincent Memorial Hospital (1950). The Cytologic Diagnosis of Cancer. Saunders, Philadelphia.

Weiss, L. (1959). Brit. J. clin. Pract., 13, 272.

\section{THE JUNE (1960) ISSUE}

The June (1960) issue contains the following papers:-

Crohn's Disease (Regional Enteritis) of the Large Intestine and its Distinction from Ulcerative Colitis. H. E. LOCKHART-MUMmerY and B. C. MORSON.

Volvulus of the Caecum. Geoffrey Chamberlain.

A Test of Pancreatic Function in Man Based on the Analysis of Duodenal Contents after Administration of Secretin and Pancreozymin. P. BuRTON, D. G. Evans, A. A. Harper, Henry T. Howat, S. Oleesky, J. E. Scott, and H. Varley.

Serum Amylase and Serum Lipase Levels in Man after Administration of Secretin and Pancreozymin. P. Burton, Elizabeth M. Hammond, A. A. Harper, Henry T. Howat, J. E. ScotT, and H. VARLEY.

Leucine Aminopeptidase with Special Reference to Ulcerative Colitis. L. J. WITTS and S. W. Perry.

Effect of Glucagon on Gastric Secretion in Man. A. G. MeLrose.

Effect of Glucagon on Gastric Secretion in the Dog. S. D. Clarke, D. W. NeILl, and R. B. WeLBOURN.

Partial Gastrectomy for Peptic Ulcer. V. S. Brookes, J. A. H. WATERhouse, and P. A. THORN.

A Report of Two Cases of Whipple's Disease Diagnosed by Peroral Small Intestinal Biopsy. C. J. Dickinson, M. Hartog, and Margot Shiner.

Hepatic Necrosis Due to Marsilid. C. F. McCarthy and M. R. Sheridan.

Smoking and the Alimentary Tract: A Review. R. S. PaCKard.

\section{Present-day Techniques}

Use of Bisacodyl in Preparation of the Bowels for a Barium Enema. J. G. SowERButTs. Gastro-intestinal Blood Loss Measured by Radioactive Chromium. A. D. CAmeron.

A number of copies are still available and may be obtained from the Publishing Manager, British Medical Association, Tavistock Square, W.C.1, price 17s. 6d. 